\title{
CARACTERISTIQUES PHYSICO-CHIMIQUES ET ORGANOLEPTIQUES DU CAFE ROBUSTA (Coffea canephora L.) EN FONCTION DES TERROIRS ET DES TECHNIQUES CULTURALES EN CÔTE D'IVOIRE
}

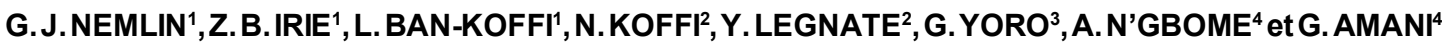 \\ ${ }^{1}$ Laboratoire de Technologie du café et du cacao, Centre National de Recherche Agronomique (CNRA), Station de \\ Recherche Technologique, 08 BP 881 Abidjan 08, Côte d'Ivoire. \\ ${ }^{2}$ Station de Recherche sur le café et le cacao, Centre National de Recherche Agronomique (CNRA), BP 808, Divo, \\ Côte d'Ivoire. \\ ${ }^{3}$ Laboratoire Central Sols, Plantes, Eaux, Centre National de Recherche Agronomique (CNRA).
}

${ }^{4}$ Unité de formation et de Recherche/Sciences et Technologies des Aliments (UFR/STA), Université d'Abobo-Adjamé, 02 BP 801, Abidjan 02, Côte d'Ivoire

\begin{abstract}
RESUME
L'étude de la qualité chimique et des caractéristiques sensorielles du café robusta a été effectuée en vue d'identifier des terroirs spécifiques de production de café robusta en Côte d'lvoire. Des cafés robusta provenant d'Aboisso, d'Abengourou et de Divo ont été analysés. Les résultats montrent que les trois régions constituent 3 terroirs distincts. A l'intérieur d'un même terroir, les niveaux d'application des techniques culturales n'ont pas eu, en général, une influence significative sur la composition du café. Par contre, une analyse factorielle discriminante a permis de montrer que le terroir de Divo est très éloigné de celui d'Aboisso, celui d'Abengourou se situe entre ceux de Divo et d'Aboisso, mais avec une variabilité plus grande. En comparant les terroirs pour chaque constituant chimique analysé, l'étude a montré que le café d'Abengourou est relativement plus riche en caféine $(2,78 \pm 0,23 \%)$ suivi de celui d'Aboisso $(2,45 \pm$ $0,19 \%)$ puis de Divo $(2,34 \pm 0,26 \%)$, plus riche en acide chlorogénique $(14,53 \pm 0,38 \%)$ suivi par celui de Divo $(13,67 \pm 0,65 \%)$ puis d'Aboisso $(13,25 \pm 0,25 \%)$. Enfin, les cafés provenant d'Abengourou et de Divo sont les plus riches en matière grasse avec respectivement $11,68 \pm 0,38 \%$ et $11,52 \pm 0,58 \%$. L'analyse sensorielle réalisée sur les différentes préparations de cafés a montré que le café robusta provenant de chaque terroir correspond à un marché spécifique dans les pays importateurs.
\end{abstract}

Mots-clés : Café robusta, terroirs, qualité chimique, caractéristiques sensorielles, Côte d'Ivoire.

\begin{abstract}
PHYSICO-CHEMICAL AND ORGANOLEPTIC CHARACTERISTICS OF ROBUSTA COFFEE (COFFEA CANEPHORA L.) ACCORDING TO TERROIRS AND FARMING TECHNICS IN COTTED'IVOIRE
\end{abstract}

The study of chemical quality and sensorial characteristics of robusta coffee has been undertaken. This work aimed at determining specific production terroirs of robusta coffee in Côte d'lvoire. Coffee samples from Aboisso, Abengourou and Divo regions have been analysed. The results showed that the three origins constitute three separate terroirs and the different levels of farming practices did not influence coffee content. However, statistical analysis showed that on the basis of the amount of the compounds analysed, the terroir of Divo was far from that of Aboisso, the terroir of Abengourou was intermediate to those of Divo and Aboisso but with a highest variability. Regarding the compounds, the comparative study of the three regions showed that coffee from Abengourou was relatively richer in caffeine $(2,78 \pm 0,23 \%)$ than that from Aboisso $(2,45$ $\pm 0,19 \%)$, followed by Divo $(2,34 \pm 0,26 \%)$. This latter was richer in chlorogenic acid $(14,53 \pm 0,38 \%)$ than Divo $(13,67 \pm 0,65 \%)$, followed by Aboisso. Coffees from Abengourou and Divo are the richest in fats. Sensorial analysis performed on the different coffee liquors showed that coffee from every region (terroir) can be sold on a specific market in consumer countries.

Key-words : Robusta coffee, terroirs, chemical quality, sensorial characteristics, Côte d'lvoire. 


\section{INTRODUCTION}

Le café robusta est une variété de l'espèce Coffea canephora qui compte plusieurs autres variétés dont seulement 5 sont comestibles (Cambroni, 1989). II représente $65 \%$ de la production de café du continent Africain, et environ $30 \%$ du café mondial (Asiedu, 1989). La plante préfère les zones climatiques chaudes très humides (Jagoret et Descroix, 2002). En Côte d'Ivoire, la culture du café robusta concerne près de 500000 planteurs et leurs familles et participe à la création de nombreux emplois dans les secteurs secondaire et tertiaire. Ainsi, plus de 2 millions de personnes vivent de l'exploitation du café robusta dans ce pays. Il est exporté à plus de $70 \%$ dans les pays consommateurs dont les critères de choix diffèrent, en particulier du point de vue des qualités gustatives et de la taille des grains (Anonyme, 2001). Les robustas d'Afrique occidentale et certains d'Afrique centrale sont particulièrement appréciés en Italie, en France, en Belgique et en Grande-Bretagne pour leur corps et leur goût dur et fort. Les robustas au goût neutre provenant du Vietnam, de l'Indonésie, de l'Ouganda et du Togo sont demandés en Allemagne et en Europe du Nord (Anonyme, 2001). De même qu'il existe des différences entre les robustas selon les provenances (pays), on trouve à l'intérieur d'un même territoire des robustas aux caractéristiques physico-chimiques différentes selon les zones de production ou terroirs (Smith, 1985).

De nombreux travaux effectués ont permis de déterminer les paramètres chimiques de la qualité du café qui contribuent à l'expression des arômes du produit torréfié et qui caractérisent des terroirs spécifiques dans un pays producteur donné. Les composés chimiques concernés sont notamment le saccharose (Trugo, 1985), la caféine (Charrier et Berthaud, 1975), la trigonelline (Mayer, 1981), l'acide chlorogénique (Clifford, 1985) et les matières grasses. En effet, les glucides dont le saccharose, représentent plus de $50 \%$ de la matière sèche du café vert. Ils sont transformés au cours de la torréfaction en composés aromatiques (Guyot et al., 1984). La caféine, substance naturelle azotée, est une triméthylxanthine du groupe des alcaloïdes qui a été aussi extraite d'autres plantes dont le thé, le cacao ou les noix de cola (Guyot et al., 1993). Elle est présente dans le café à différentes teneurs selon les variétés : de $0,9 \%$ à $1,6 \%$ dans l'arabica et de $1,5 \%$ à $2,9 \%$ dans le robusta (Clarke et Macrae, 1985). La caféine est un produit stimulant du système nerveux central qui contribue à $10 \%$ à l'amertume du café à la tasse (Voilley et al., 1977). La trigonelline est, comme la caféine, un autre alcaloïde du café, mais présente en quantités plus faibles (0,2 à $1 \%)$ (Coste, 1989). Elle contribue à l'amélioration de l'arôme du café, car elle est transformée lors de la torréfaction en d'autres composés secondaires, dont l'acide nicotinique, qui est assimilé par l'organisme sous forme de vitamine B (Clarke et Macrae, 1985). Les acides chlorogéniques, combinaisons de l'acide cinnamique et ses dérivés avec l'acide quinique, sont les polyphénols les plus abondants et sont à l'origine de la grande variabilité des antioxydants contenus dans le café (Svilaas, 2004). Les teneurs des acides chlorogéniques diffèrent de façon très significative entre les deux espèces cultivées: 5 - $7,5 \%$ dans l'arabica et $7-10 \%$ dans le robusta (Clarke et Macrae, 1985). Malgré des propriétés antioxydantes (anti-radicaux libres), leur présence dans le café n'est pas particulièrement appréciée sur le plan sensoriel, car ils contribuent à l'astringence et à l'amertume du café boisson obtenue après torréfaction (Amorim et al., 1973 ; Haslam, 1981). Avec les triglycérides et les diterpènes en tête, les matières grasses sont présentes en quantité appréciable dans les grains de café robusta (7 à $13 \%$ ). Ils sont à l'origine de l'acidité fine, dont on estime qu'elle améliore la qualité des cafés (Picard et al., 1984).

Compte tenu des exigences du marché international en terme de qualité et de compétitivité, la nécessité de trouver des voies de valorisation du café robusta de Côte d'Ivoire par l'utilisation optimale des terroirs devient une préoccupation majeure, tant pour les producteurs, que pour les partenaires de ce secteur. La présente étude vise à identifier des terroirs spécifiques de production de café robusta en Côte d'Ivoire sur la base des caractéristiques physico-chimiques du café vert et du profil sensoriel du café torréfié.

\section{MATERIEL ET METHODES}

\section{MILIEUXD’ETUDE}

Trois terroirs ont été sélectionnés en fonction du climat, des types de sols et du tonnage de café produit dans ces zones. Il s'agit de la région d'Abengourou, avec des sols issus de schistes 
et une production de $13 \%$ de café ; la région d'Aboisso sur des sols tertiaires avec une production de $14 \%$; la région de Divo sur des sols granitiques avec une production de $14 \%$. L'ensemble des 3 zones représente ainsi $41 \%$ de la production nationale du café en Côte d'lvoire.

\section{MATERIEL VEGETAL}

En fonction du tonnage de café produit sur chaque parcelle (entre 1 et 2 tonnes), 40 échantillons élémentaires de $100 \mathrm{~g}$ chacun, soit $4 \mathrm{~kg}$ constituant l'échantillon global ont été prélevés, selon la norme n $4001 / 2006$ de l'Union Européenne.

A l'intérieur de chaque terroir, les producteurs ont été choisis en fonction de 3 niveaux d'application des techniques culturales (Anonyme, 1999):

- le niveau 1 correspond aux paysans ayant utilisé un matériel végétal sélectionné et une parcelle entretenue selon les normes recommandées par la recherche ;

- le niveau 2 correspond aux paysans ayant utilisé un matériel végétal sélectionné, mais qui n'ont pas entretenu leur parcelle ;

- le niveau 3 correspond aux paysans ayant utilisé du matériel tout venant non sélectionné et ayant entretenu ou non leur parcelle.

Dans chaque terroir, 3 producteurs par niveau ont été choisis au hasard, soit au total 27 producteurs pour les 3 terroirs.

\section{METHODES D'ANALYSE}

A partir de chaque échantillon global de $4 \mathrm{~kg}$, $200 \mathrm{~g}$ de café vert ont été broyés et ont servi d'échantillon de laboratoire pour la détermination des principaux paramètres de la qualité chimique du café.

Les taux d'humidité ont été déterminés sur $5 \mathrm{~g}$ de café en poudre par séchage à $105^{\circ} \mathrm{C}$ pendant 24 heures dans une étuve de type Memmert, $A$ 0557, No 596020, Western Germany (AOAC, 1997).

Les cendres ont été dosées selon la méthode A.O.A.C (1997). Elle a consisté à incinérer $5 \mathrm{~g}$ de café en poudre à $550{ }^{\circ} \mathrm{C}$, pendant 8 heures dans un four à moufle (VOLCA V 50, Prolabo, Paris, France).
L'acidité totale a été déterminée sur $10 \mathrm{~g}$ de café en poudre à l'aide d'une solution de soude à $0,1 \mathrm{~N}$ en présence de phénophtaléine comme indicateur coloré.

Les teneurs en sucres réducteurs ont été obtenues sur $5 \mathrm{~g}$ de café en poudre par la méthode à l'acide 3,5-dinitro-salicylique (Bernfeld, 1955). La détermination de la teneur en protéines a été effectuée par la méthode de Kjeldahl par dosage de l'azote total contenu dans $1 \mathrm{~g}$ d'échantillon. Le coefficient de conversion de l'azote en protéines utilisé est 6,25 . La matière grasse a été extraite de $3 \mathrm{~g}$ d'échantillon par l'hexane, le solvant éliminé et le résidu obtenu séché à l'étuve pendant $24 \mathrm{~h}$, puis pesé.

La teneur en caféine a été déterminée sur $0,5 \mathrm{~g}$ de café en poudre par la méthode de Trugo et al. (1983). La caféine a été extraite du café par l'eau à $100{ }^{\circ} \mathrm{C}$ et décomplexée par l'addition d'oxyde de magnésium. Elle a été séparée et identifiée par HPLC et quantifiée par un détecteur ultraviolet à $278 \mathrm{~nm}$.

L'acide chlorogénique a été dosé par la méthode de Carelli et al. (1974). Il a été extrait de $1 \mathrm{~g}$ de café vert en poudre par du méthanol à $70 \%$. L'extrait a été purifié sur une colonne de poudre de polyamide, l'acide chlorogénique a été ensuite élué par du méthanol basique et mesuré au spectrophotomètre à $324 \mathrm{~nm}$.

La teneur en trigonelline a été déterminée par la méthode de Trugo et al. (1983). La trigonelline a été extraite de $0,6 \mathrm{~g}$ de café par l'eau à $100^{\circ} \mathrm{C}$ et décomplexée par l'addition d'oxyde de magnésium. Elle a été séparée et identifiée par HPLC et quantifiée par un détecteur ultraviolet à $266 \mathrm{~nm}$.

Le profil sensoriel des différents échantillons a été déterminé à l'aide d'une analyse descriptive quantitative (Langler et al., 1967 ; Amorim et al., 1973). Le panel était composé de 9 personnes, formées à la détection des saveurs de base et à l'analyse sensorielle du café, à l'Organisation Interafricaine de café (OIAC) à Abidjan.

Les analyses statistiques des différentes données ont été réalisées à l'aide du logiciel XLSTAT 7.5.3. Une analyse de variance à deux critères (ANOVA 2) a été effectuée. Pour chaque paramètre, les valeurs moyennes ont été comparées selon les niveaux d'applications des 
techniques culturales et selon les terroirs. Le niveau de signification a été évalué au seuil de $5 \%$. Une analyse en composantes principales (ACP) et une analyse factorielle discriminante (AFD) ont été effectuées afin de mettre en évidence une structure de groupe selon les terroirs, et les distances de Mahalanobis exprimant l'éloignement ou la proximité des terroirs les uns par rapport aux autres.

\section{RESULTATS}

TAUX D'HUMIDITE, CENDRES, SUCRES RÉDUCTEURS, MATIERE GRASSE ET ACIDITE TOTALE DU CAFE EN FONCTION DES TERROIRS

On note une différence significative $(P<0,05)$ entre les taux d'humidité du café des terroirs d'Abengourou, d'Aboisso et de Divo (Tableau 1).
Le café provenant d'Abengourou présente une teneur moyenne en humidité plus basse $(10,70$ $\pm 0,46 \%)$ que ceux plantés à Aboisso $(11,01 \pm$ $0,70 \%)$ et Divo $(11,20 \pm 0,72 \%)$. Les teneurs en cendres varient selon les terroirs. Elles sont de $4,16 \pm 0,34 \% ; 3,71 \pm 0,37 \%$ et 3,77 $\pm 0,31 \%$, respectivement, pour les terroirs d'Abengourou, d'Aboisso et de Divo. L'examen des taux de sucres réducteurs, montre qu'il n'y a pas eu de différence significative entre les terroirs. Ils sont de $1,43 \pm 0,43 \% ; 1,38 \pm$ $0,32 \%$ et $1,25 \pm 0,15 \%$, respectivement, à Abengourou, Aboisso et Divo. On note une teneur élevée en matière grasse dans le café d'Abengourou $(11,68 \pm 0,38 \%)$ par rapport à celle du café des terroirs de Divo (11,52 \pm $0,58 \%$ ) et d'Aboisso $(10,91 \pm 0,47 \%)$. L'acidité totale du café du terroir d'Aboisso est plus élevée avec $97,81 \pm 2,55 \mathrm{mEq}-\mathrm{g} / 100 \mathrm{~g}$ de matière sèche que ceux d'Abengourou $(97,02 \pm 1,07 \mathrm{mEq}-\mathrm{g} /$ $100 \mathrm{~g})$ et Divo $(87,07 \pm 1,80 \mathrm{mEq}-\mathrm{g} / 100 \mathrm{~g})$.

Tableau 1 : Taux d'humidité, cendre, sucres réducteurs, lipides et acidité totale de café des 3 terroirs.

Moisture content, ashes, reducing sugars, lipids and total acidity of coffee of the three terroirs.

\begin{tabular}{lccccc}
\hline Terroirs & $\begin{array}{c}\text { Humidité } \\
(\%)\end{array}$ & $\begin{array}{c}\text { Cendre } \\
(\%)\end{array}$ & $\begin{array}{c}\text { Sucres réducteurs } \\
(\%)\end{array}$ & $\begin{array}{c}\text { Lipide } \\
(\%)\end{array}$ & $\begin{array}{c}\text { Acidité totale } \\
(\mathrm{mEq}-\mathrm{g} / 100 \mathrm{~g})\end{array}$ \\
\hline Abengourou & $10,7 \pm 0,46^{\mathrm{a}}$ & $4,16 \pm 0,34^{\mathrm{b}}$ & $1,43 \pm 0,43^{\mathrm{a}}$ & $11,68 \pm 0,38^{\mathrm{a}}$ & $97,02 \pm 1,07^{\mathrm{a}}$ \\
Aboisso & $11,01 \pm 0,7^{\mathrm{ab}}$ & $3,71 \pm 0,37^{\mathrm{a}}$ & $1,38 \pm 0,32^{\mathrm{a}}$ & $10,91 \pm 0,47^{\mathrm{b}}$ & $97,81 \pm 2,55^{\mathrm{a}}$ \\
Divo & $11,2 \pm 0,72^{\mathrm{b}}$ & $3,77 \pm 0,31^{\mathrm{ab}}$ & $1,25 \pm 0,15^{\mathrm{a}}$ & $11,52 \pm 0,58^{\mathrm{b}}$ & $87,07 \pm 1,80^{\mathrm{a}}$ \\
Moyenne & $10,97 \pm 0,62$ & $3,88 \pm 0,34$ & $1,35 \pm 0,3$ & $11,37 \pm 0,47$ & $93,96 \pm 1,80$ \\
\hline
\end{tabular}

Les valeurs affectées de différentes lettres sont significativement différentes au seuil de $5 \%$.

TENEUR EN PROTEINES, ACIDE CHLOROGENIQUE, CAFEINEET TRIGONELLINE DANS LES DIFFERENTS TERROIRS

Les taux de protéine des terroirs d'Abengourou $(13,92 \pm 1,35 \%)$, Aboisso (14,99 $\pm 1,81 \%)$ et Divo $(15,35 \pm 2,97 \%)$ ne sont pas significativement différents au seuil de $5 \%$ (Figure 1). Les teneurs en acide chlorogénique sont par contre différentes $(P<0,05)$ dans les terroirs d'Abengourou (14,53 $\pm 0,38 \%)$, Aboisso $(13,25 \pm 0,25 \%)$ et Divo $(13,67 \pm 0,65 \%)$, de même que celles de la caféine qui sont respectivement $2,78 \pm 0,23 \% ; 2,45 \pm 0,19 \%$ et 2,34 $\pm 0,26 \%$ à Abengourou, Aboisso et Divo. II n'y a pas eu d'effet significatif du terroir au seuil de $5 \%$, sur la teneur en trigonelline du café robusta issu des terroirs d'Abengourou $(0,7 \pm 0,06 \%)$, Aboisso $(0,67 \pm 0,02 \%)$ et Divo $(0,69 \pm 0,03 \%)$.

La représentation des échantillons de café sur les 2 premiers plans principaux (Figure 2) de l'ACP met en évidence une structure de groupe selon les terroirs : Divo d'un côté et Aboisso et une grande partie des échantillons d'Abengourou de l'autre côté. L'analyse factorielle discriminante a permis de se rendre compte des distances de Mahalanobis (Tableau 2) exprimant l'éloignement ou la proximité des terroirs les uns par rapport aux autres. Sur la base des composés chimiques analysés, le terroir de Divo est très éloigné de celui d'Aboisso, alors que le terroir d'Abengourou se situe entre ceux de Divo et d'Aboisso (Figure 3). 


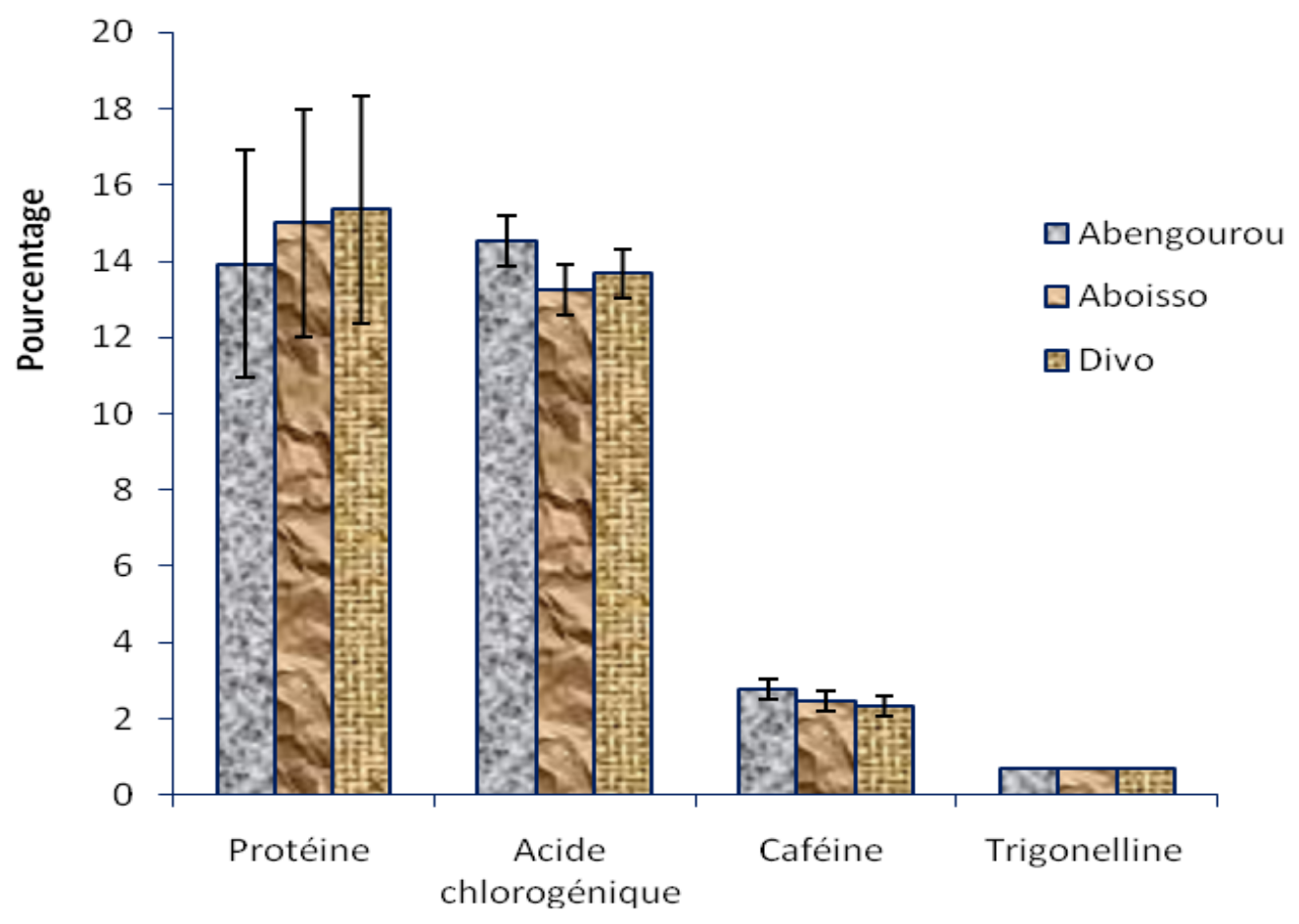

Figure 1 : Taux de protéines, acide chlorogénique, caféine et trigonelline du café des 3 terroirs.

Proteins, chlorogenic acid, caffeine and trigonelline content of coffee of the three terroirs.

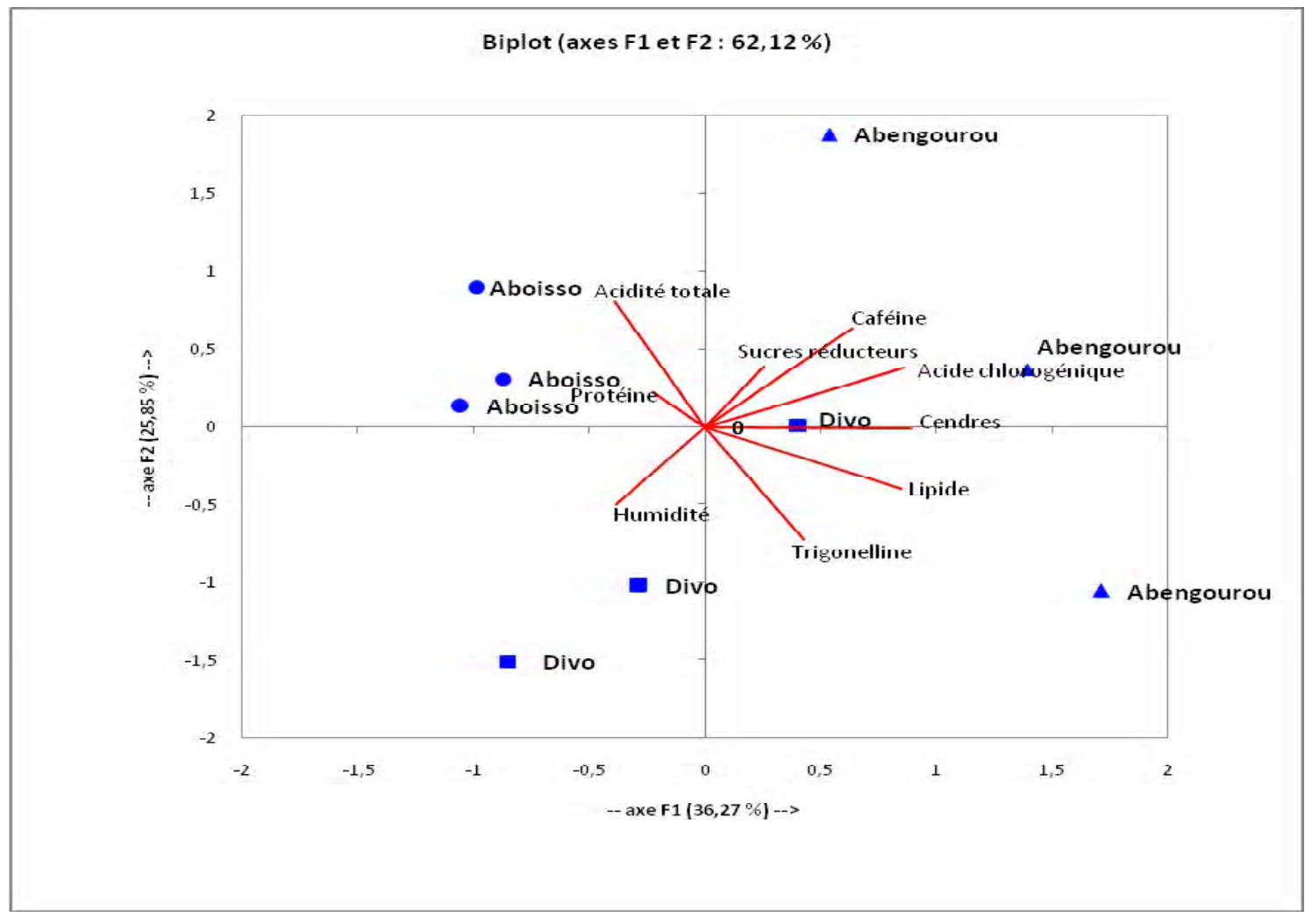

Figure 2 : Variation de la composition physico-chimique des échantillons selon les terroirs. Variation of the physico-chemical composition of samples from terroirs. 
Tableau 2 : Carrés des distances de Mahalanobis entre les terroirs. Square of Mahalanobis distances between terroirs.

\begin{tabular}{lccc}
\cline { 2 - 4 } & \multicolumn{3}{c}{ Distance de Mahalanobis } \\
\cline { 2 - 4 } Abengourou & Abengourou & Aboisso & Divo \\
\cline { 2 - 4 } & 0 & 14,209 & 5,508 \\
\hline Aboisso & 14,209 & 0 & 6,048 \\
\hline Divo & 5,508 & 6,048 & 0 \\
\hline
\end{tabular}

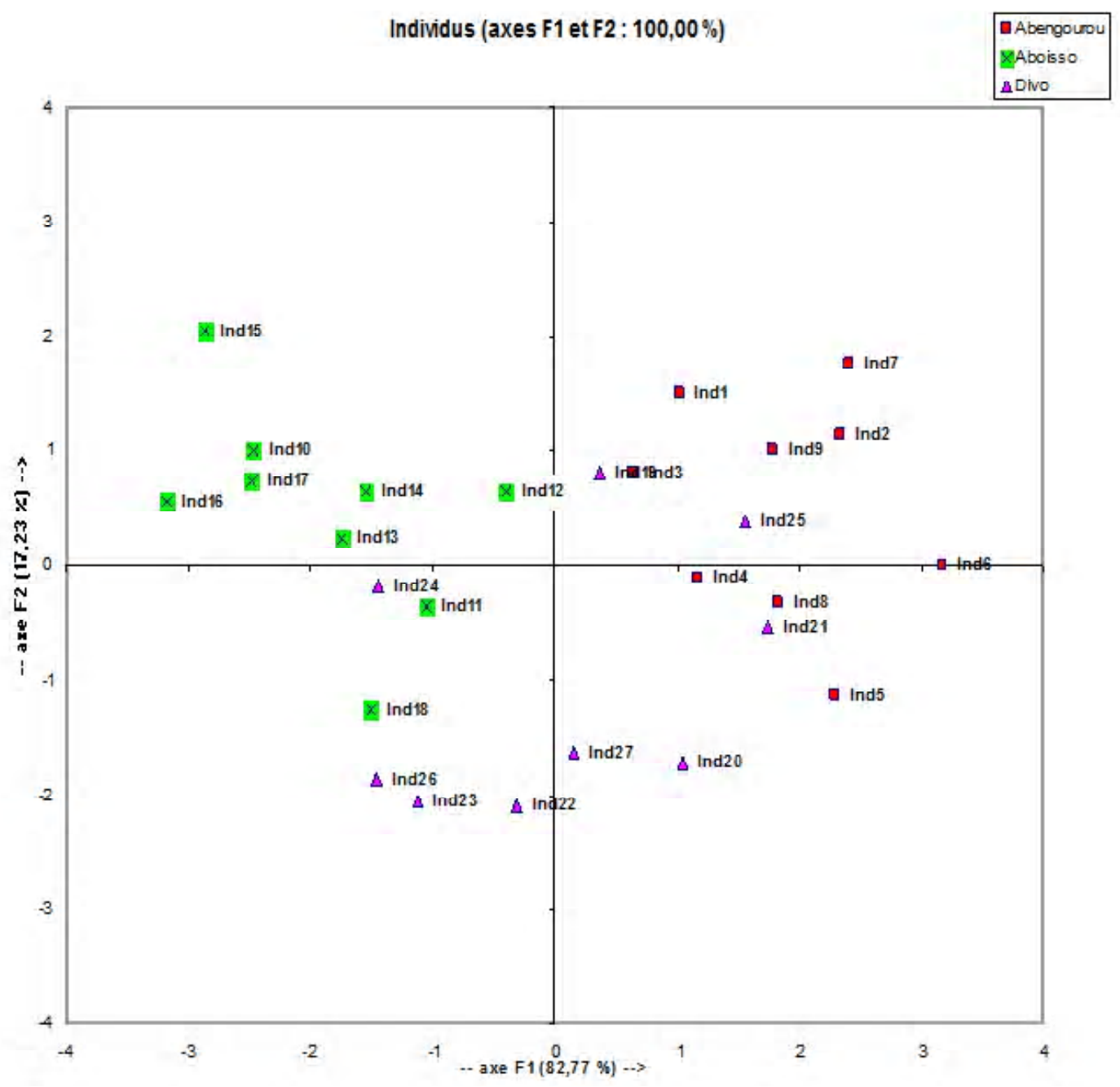

Figure 3 : Représentation des échantillons selon les deux premières fonctions discriminantes. Representation of samples according to the first two discriminent functions. 
TAUXD'HUMIDITE, DE CENDRE, DE SUCRES RÉDUCTEURS, DE MATIERE GRASSE ET D'ACIDITE TOTALE DU CAFE EN FONCTION DES NIVEAUX D'APPLICATIONS DES TECHNIQUES CULTURALES DANS CHAQUE TERROIR

Les taux d'humidité du café ont été de $10,69 \% ; 10,51 \%$ et $10,87 \%$, respectivement, pour les niveaux 1 , 2 et 3 d'Abengourou (Tableau $3)$. On note une différence significative entre le niveau 3 et les niveaux 1 et 2 de ce terroir. A Aboisso, le niveau 2 présente le taux d'humidité le plus élevé, avec $11,48 \%$ alors que le niveau 1 (10,51\%) et le niveau $3(10,75 \%)$ possèdent sensiblement les mêmes taux d'humidité. Les niveaux des terroirs de Divo n'ont révélé aucune différence significative entre ces taux d'humidité. L'analyse des valeurs de cendres a montré qu'il n'y a pas eu de différence significative $(P>0,05)$ entre les niveaux des terroirs d'Aboisso et Divo, alors qu'il y a une différence significative entre les niveaux d'Abengourou. Concernant les teneurs en sucres réducteurs, en lipides et en acidité totale, aucune différence significative $(P>0,05)$ n'a été observée entre les niveaux de techniques culturales quelle que soit la région.

Tableau 3 : Taux d'humidité, cendres, sucres réducteurs, lipides et acidité totale du café en fonction des niveaux N1, N2 et N3 des techniques culturales.

Moisture, ashes, reducing sugars, lipids and total acidity content of coffee according to levels N1, N2, N3 of farming techniques.

\begin{tabular}{|c|c|c|c|c|c|}
\hline Niveaux & $\begin{array}{c}\text { Humidité } \\
(\%)\end{array}$ & $\begin{array}{c}\text { Cendre } \\
(\%)\end{array}$ & $\begin{array}{c}\text { Sucre réducteur } \\
(\%)\end{array}$ & $\begin{array}{c}\text { Lipide } \\
(\%)\end{array}$ & $\begin{array}{l}\text { Acidité totale } \\
\text { (mEq-g/100g) }\end{array}$ \\
\hline N1 Abengouro & $10,69 \pm 0,28^{a}$ & $3,84 \pm 0,36^{a}$ & $1,54 \pm 0,76^{a}$ & $11,25 \pm 0,39^{a}$ & $96,40 \pm 13,43^{a}$ \\
\hline N2 Abengourou & $10,51 \pm 0,5^{a}$ & $4,39 \pm 0,43^{b}$ & $1,29 \pm 0,41^{a}$ & $11,87 \pm 0,54^{a}$ & $94,43 \pm 13,11^{a}$ \\
\hline N3 Abengourou & $10,87 \pm 0,35^{b}$ & $4,25 \pm 0,07^{a b}$ & $1,47 \pm 0,09^{a}$ & $11,91 \pm 0,15^{a}$ & $92 \pm 5,05^{a}$ \\
\hline N1 Aboisso & $10,51 \pm 0,34^{a}$ & $3,75 \pm 0,44^{a}$ & $1,2 \pm 0,62^{a}$ & $10,81 \pm 0,46^{a}$ & $97,83 \pm 5,31^{a}$ \\
\hline N2 Aboisso & $11,48 \pm 0,95^{b}$ & $3,78 \pm 0,33^{a}$ & $1,25 \pm 0,26^{a}$ & $11,07 \pm 0,62^{a}$ & $97,28 \pm 8,34^{a}$ \\
\hline N3 Aboisso & $10,75 \pm 0,71^{a}$ & $3,58 \pm 0,35^{a}$ & $1,69 \pm 0,23^{a}$ & $10,84 \pm 0,65^{a}$ & $94,47 \pm 4,14^{a}$ \\
\hline N1 Divo & $10,76 \pm 0,50^{a}$ & $3,93 \pm 0,16^{a}$ & $1,4 \pm 0,06^{a}$ & $11,65 \pm 0,77^{\mathrm{a}}$ & $93,55 \pm 6,66^{a}$ \\
\hline N2 Divo & $11,04 \pm 0,29^{a}$ & $3,69 \pm 0,13^{a}$ & $1,19 \pm 0,23^{a}$ & $11,25 \pm 0,37^{a}$ & $92,01 \pm 2,05^{a}$ \\
\hline N3 Divo & $11,25 \pm 0,93^{a}$ & $3,7 \pm 0,38^{a}$ & $1,17 \pm 0,18^{a}$ & $11,66 \pm 0,28^{a}$ & $92,64 \pm 8,15^{a}$ \\
\hline
\end{tabular}

Les valeurs affectées de différentes lettres sont significativement différentes au seuil de $5 \%$.

TENEUR EN PROTEINES, ACIDE CHLOROGENIQUE, CAFEINE, TRIGONELLINE EN FONCTION DES NIVEAUX D'APPLICATIONS DES TECHNIQUES CULTURALES DANS CHAQUE TERROIR

Pour les teneurs en protéines, aucun effet significatif n'a été observé concernant les différents niveaux d'application des techniques culturales dans les terroirs d'Abengourou et d'Aboisso (Tableau 4). Les taux de protéines trouvés dans le café provenant des niveaux $1 ; 2$ et 3 d'Abengourou ont été respectivement de $14,57 \% ; 13,9 \%$ et $13,28 \%$. Ceux d'Aboisso ont été respectivement de $15,56 \% ; 15,36 \%$ et $14,06 \%$. Enfin, pour le terroir de Divo, les niveaux $1 ; 2$ et 3 ont des teneurs en protéine de $16,68 \% ; 12,75 \%$ et $16,62 \%$, le niveau 2 ayant présenté le taux le plus bas.
La détermination des teneurs en acide chlorogénique fait apparaître qu'il n'y a pas eu de différence significative entre les niveaux des terroirs d'Abengourou et d'Aboisso, par contre, il y a eu une différence entre le niveau 1 de Divo avec $14,19 \%$ et les niveaux 2 et 3 avec respectivement $12,99 \%$ et $13,82 \%$.

L'évolution de la teneur en caféine montre des différences non significatives au seuil de $5 \%$, entre les niveaux des trois terroirs d'Abengourou, Aboisso et Divo.

Pour les teneurs en trigonelline, les valeurs des 3 niveaux du terroir d'Abengourou ont été significativement différentes. Le pourcentage du niveau 3 a été de $0,77 \%$, alors que ceux du niveau 1 et 2 ont été de l'ordre de $0,64 \%$ et $0,67 \%$. 
Tableau 4 : Taux de protéines, acide chlorogénique, caféine et trigonelline en fonction des niveaux N1, N2 et N3 des techniques culturales.

Proteins, chlorogenic acid, caffeine and trigonelline content of coffee according to levels N1, N2, N3 of farming techniques.

\begin{tabular}{lcccc}
\hline Niveaux & Protéine (\%) & Acide chlorogénique (\%) & Caféine (\%) & Trigonelline (\%) \\
\hline N1 Abengourou & $14,57 \pm 1,28^{a}$ & $14,73 \pm 0,26^{a}$ & $2,99 \pm 0,17^{a}$ & $0,64 \pm 0,01^{a}$ \\
N2 Abengourou & $13,9 \pm 1,42^{a}$ & $14,6 \pm 0,29^{a}$ & $2,63 \pm 0,19^{a}$ & $0,67 \pm 0,02^{a}$ \\
N3 Abengourou & $13,28 \pm 1,57^{a}$ & $14,25 \pm 0,51^{a}$ & $2,74 \pm 0,24^{a}$ & $0,77 \pm 0,03^{\mathrm{a}}$ \\
\hline N1 Aboisso & $15,56 \pm 2,24^{\mathrm{a}}$ & $13,38 \pm 0,34^{\mathrm{a}}$ & $2,44 \pm 0,24^{\mathrm{a}}$ & $0,67 \pm 0,02^{\mathrm{a}}$ \\
N2 Aboisso & $15,36 \pm 1,39^{\mathrm{a}}$ & $13,29 \pm 0,18^{\mathrm{a}}$ & $2,53 \pm 0,15^{\mathrm{a}}$ & $0,67 \pm 0,06^{\mathrm{a}}$ \\
N3 Aboisso & $14,06 \pm 2,09^{\mathrm{a}}$ & $13,08 \pm 0,16^{\mathrm{a}}$ & $2,4 \pm 0,23^{\mathrm{a}}$ & $0,68 \pm 0,02^{\mathrm{a}}$ \\
\hline N1 Divo & $16,68 \pm 2,25^{\mathrm{a}}$ & $14,19 \pm 0,04^{\mathrm{a}}$ & $2,5 \pm 0,16^{\mathrm{a}}$ & $0,69 \pm 0,04^{\mathrm{a}}$ \\
N2 Divo & $12,75 \pm 1,5^{\mathrm{a}}$ & $12,99 \pm 0,02^{\mathrm{a}}$ & $2,12 \pm 0,13^{\mathrm{a}}$ & $0,69 \pm 0,04^{\mathrm{a}}$ \\
N3 Divo & $16,62 \pm 3,5^{\mathrm{a}}$ & $13,82 \pm 0,75^{\mathrm{a}}$ & $2,39 \pm 0,36^{\mathrm{a}}$ & $0,7 \pm 0,02^{\mathrm{a}}$ \\
\hline
\end{tabular}

Les valeurs affectées de différentes lettres sont significativement différentes au seuil de $5 \%$

\section{CARACTERISTIQUES SENSORIELLES DU CAFE ROBUSTA}

Le café du terroir d'Aboisso exhale un arôme caractéristique plus faible que celui d'Abengourou et Divo. Quant à la qualité aromatique, le café du terroir d'Aboisso et celui d'Abengourou ont eu une note supérieure à 2 sur une échelle de notation linéaire et structurée allant de 0 à 5 , tandis que celui de Divo a eu une note inférieure. De plus, à Aboisso, le café a présenté une acidité nettement supérieure, mais avec une amertume inférieure aux autres terroirs. Le café d'Abengourou et celui de Divo ont été les plus astringents. Les panélistes ont préféré le café des terroirs d'Aboisso et d'Abengourou (Figure 4).

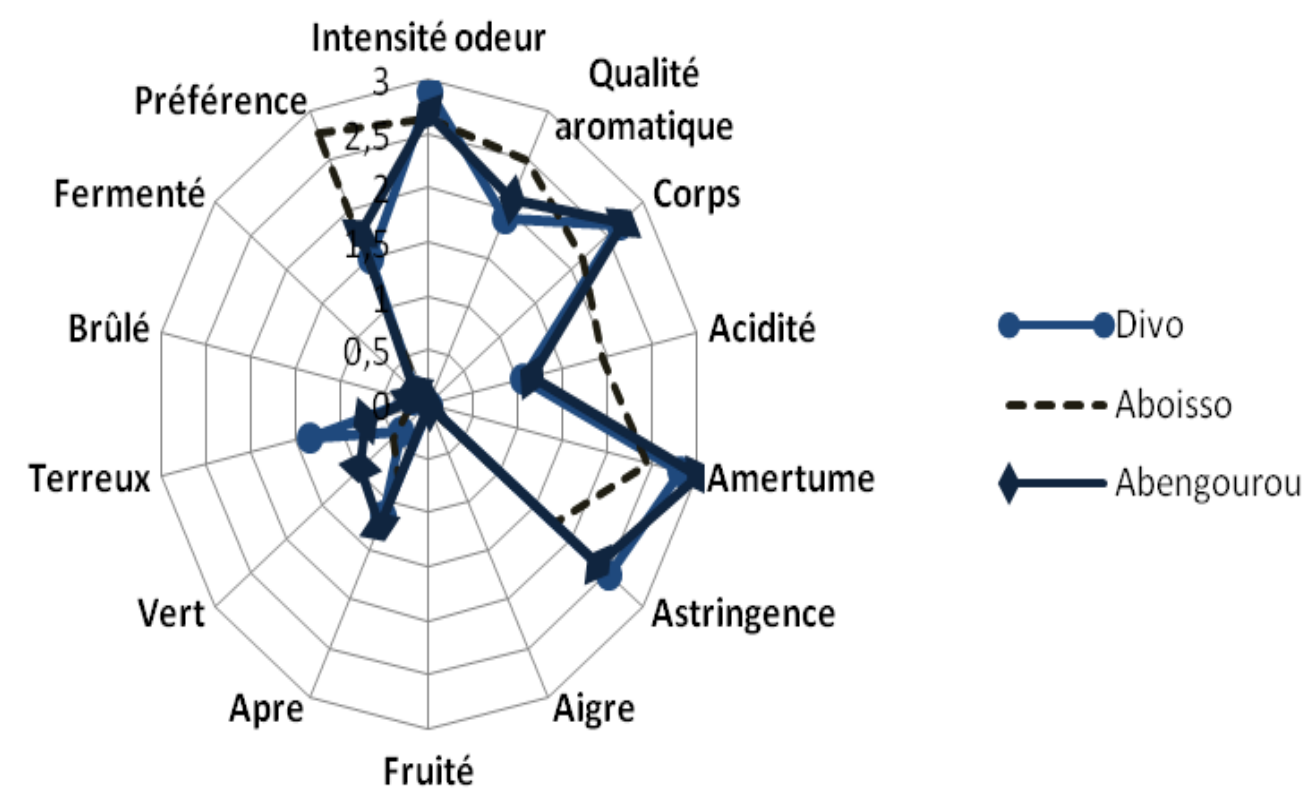

Figure 4 : Profil sensoriel du café robusta des différents terroirs.

Sensorial profile of robusta coffee of the different terroirs. 


\section{DISCUSSION}

Les taux d'humidité des cerises fraîches du café robusta sont liés au pourcentage de matière sèche constituée de matières minérales et de matières organiques ou matières volatiles sèches (MVS). Les concentrations en matières minérales et en MVS diffèrent selon les terroirs et les clones de robusta qui y ont été vulgarisés par la recherche (Anonyme, 1995). La variation du taux de cendres entre les terroirs est essentiellement due à la variation du taux de matières minérales et plus particulièrement du taux de potassium, élément constitutif majeur du café robusta. Cet effet du terroir sur la composition minérale du robusta avait été mis en évidence par Coste (1989), Clifford (1985) et Clarke et Walker (1974) sur du café robusta d'Ouganda et d'Angola. Pour ces auteurs, la teneur en cendres des cafés variait entre 3 et $4 \%$ selon les terroirs et elles renfermaient principalement des éléments comme $\mathrm{K}, \mathrm{Na}, \mathrm{Ca}$, $\mathrm{Mg}, \mathrm{P}$ et $\mathrm{S}$ et de nombreux oligo-éléments tels que $\mathrm{Fe}, \mathrm{Al}, \mathrm{Cu}, \mathrm{I}, \mathrm{F}, \mathrm{B}$ et $\mathrm{Mn}$.

Il a été montré que les teneurs en sucres réducteurs du café ne sont pas significativement différentes au sein d'un même terroir et dans les 3 terroirs. Ceci pourrait s'expliquer par le fait que les cerises de café ont été récoltées dans les 3 terroirs sensiblement aux mêmes stades de maturité. En effet, les sucres réducteurs qui ont migré en faibles quantités à l'intérieur de l'endocarpe sont constitués essentiellement de glucose et de fructose, issus de la dégradation enzymatique du saccharose empruntant la voie glycolytique et la voie des pentoses phosphates (Coutouly et al., 1991). La concentration en saccharose dans le café est fortement corrélée au stade de maturité des cerises. Ces observations sont proches de celles de Coste (1989). Selon cet auteur, le café vert renferme des quantités variables de sucres réducteurs, surtout le glucose dans l'ordre de $1 \%$. Leur abondance est liée au stade de maturité des cerises. Après la torréfaction, les sucres réducteurs résiduels contribuent à l'atténuation de l'amertume du café boisson (Picard et al., 1984).

Les lipides du café vert sont composés d'huile de café contenue dans l'endosperme et une petite quantité de cire localisée essentiellement dans les couches externes du grain. Ils sont constitués d'acides gras, de triglycérides, d'esters d'alcools diterpéniques, de stérols et d'esters de stérols, de tocophérols, de phosphatides et de dérivés de la tryptamine (Mayer, 1981). Les différences des taux de matière grasse observées entre les terroirs pourraient être mises en rapport avec la biosynthèse de ces différents constituants dans le café vert qui s'effectue par des processus enzymatiques complexes impliquant l'acyl-CoA et régulés par le $\mathrm{pH}$ et l'activité de l'eau (Hulme et al., 1968).

Le café du terroir d'Aboisso présente une acidité totale plus élevée que ceux d'Abengourou et de Divo. Ces résultats pourraient s'expliquer par la plus grande hétérogénéité de mélange de clones cultivés dans ce terroir, par la nature du sol et par la spécificité des pratiques post-récolte, tout particulièrement des conditions de séchage et de conservation du café (Touré, 2004). Du point de vue organoleptique, l'acidité totale est un critère de qualité recherché dans le café car il contribue fortement à relever le goût à la tasse (Perriot et Fabienne, 2006).

Les teneurs en protéines brutes dans les trois terroirs sont très élevées. Elles sont nettement supérieures au taux moyen de protéines contenues dans les robustas en général (Clarke et Macrae, 1985). Ce résultat serait dû à une combinaison de facteurs aussi bien génétiques qu'environnementaux, en rapport avec les clones vulgarisés, l'état de maturation des cerises, les pratiques culturales et les conditions écologiques. La présence de protéines dans le café des différents terroirs à des taux si élevés constitue un avantage comparatif, car les protéines améliorent la qualité organoleptique des cafés. En effet, les produits de dégradation des protéines sont responsables du développement de certains arômes du café lors de la torréfaction.

La caféine, la trigonelline et l'acide chlorogénique sont des métabolites libérés par le caféier dans le but de se défendre contre les agressions extérieures. Leur teneur peut donc varier non seulement en fonction des clones mais aussi en fonction des conditions environnementales. La teneur en caféine du café du terroir d'Abengourou est plus élevée que celles des terroirs d'Aboisso et Divo. Le niveau de caféine dans le café est diversement apprécié (Guyot et al., 1993). Une teneur élevée de caféine dans le café est importante car elle entraînerait un accroissement de la vigilance et une réduction de la fatigue. La caféine aiderait aussi à corriger les lenteurs intellectuelles et améliorerait les 
capacités de mémorisation (Warburton, 1995). Tandis que pour Frankenhauser (1986) la caféine est un excitant qui augmente le stress et favorise l'insomnie. Les taux de trigonelline des robustas provenant des 3 terroirs ne sont pas significativement différents. II en est de même pour les différents niveaux d'application des techniques culturales dans ces terroirs excepté celui du niveau 3 du terroir d'Abengourou où ce taux est différent de celui des deux autres niveaux. La trigonelline contribue à améliorer la saveur et les arômes distinctifs du café. On note une différence significative entre les trois terroirs pour ce qui concerne la teneur en acide chlorogénique, le terroir d'Abengourou en étant le plus riche. Le café en grains est l'une des sources alimentaires les plus riches en acide chlorogénique et peut apporter jusqu'à $70 \%$ de polyphénols totaux de l'alimentation. Par contre selon Claudine et Alexandre (2006), sur le plan sensoriel, la présence de cet acide dans le café vert n'est pas particulièrement appréciée car il contribue à l'astringence et à l'amertume du caféboisson obtenu après torréfaction.

Sur le plan de la qualité gustative, les intensités de certains attributs sensoriels diffèrent nettement d'un terroir à l'autre. Perriot et Fabienne (2006) ont montré que l'arôme, la saveur et le goût de la tasse de café dépendent d'une multitude de facteurs qui sont entre autres: les effets du milieu, les pratiques culturales, le mélange de différentes origines, la torréfaction, la méthode de préparation de la boisson, etc. Un changement dans cette chaîne complexe entraîne des variations de goût. Selon Barel et Jacquet (1994), les origines génétiques et géographiques d'un café lui confèrent des caractères physiques, chimiques et organoleptiques spécifiques. Les soins apportés à sa préparation et à son usinage jouent un rôle déterminant sur leur expression finale.

Ainsi, le café robusta issu des terroirs d'Aboisso et d'Abengourou a-t'-il montré un potentiel aromatique très apprécié par les panélistes. De plus, Aboisso a une acidité nettement au dessus, avec une amertume inférieure aux autres terroirs. Le café provenant de chaque terroir possède des atouts particuliers qu'il est possible de valoriser dans les pays consommateurs (Anonyme, 2001).

\section{CONCLUSION}

Cette étude a révélé une influence des zones géographiques sur les qualités physicochimiques et sensorielles du café robusta. Trois terroirs spécifiques de production de café ont été mis évidence. Le café vert provenant du terroir d'Abengourou présente des teneurs en matière grasse, acide chlorogénique et caféine plus élevées. Le terroir d'Aboisso se caractérise par des teneurs plus faibles en matière grasse, en acide chlorogénique et en trigonelline. Le café du terroir de Divo présente un taux de caféine plus faible, et un taux de protéine plus élevé. Sur le plan sensoriel, les cafés des terroirs d'aboisso et d'Abengourou ont été plus appréciés par les dégustateurs. Toutefois, le café provenant de chaque terroir peut être proposé à un marché particulier dans les pays consommateurs: le café issu du terroir de Divo est spécifique au marché du Moyen-Orient ; celui d'Aboisso constitue un café gourmet avec des débouchés sur plusieurs pays consommateurs; le café d'Abengourou convient bien au marché de l'Europe de l'Est.

Cette étude doit être étendue à toutes les zones de production de café pour l'identification et l'utilisation optimale des différents terroirs de Côte d'Ivoire.

\section{REFERENCES}

Amorim H. V., Malavolta E., Teixeira A. A., Cruz V. F., Melo M., Guercio M. A., Fossa E., Breviglieri O., Ferrari S. E. and D. M. Silva. 1973. Proc. $6^{\text {th }}$ Coll. ASIC, $113-127$.

Anonyme. 1995. Formation des techniciens spécialisés en cultures pérennes. Cahier du Stagiaire, CNRA, $87 \mathrm{p}$.

Anonyme. 1999. Techniques pour améliorer la production du café Robusta en Côte d'Ivoire. Le CNRA en 1999, p 7 - 9.

Anonyme. 2001. Caractéristiques de la demande de cafés robusta en Europe. Fonds Commun pour les Produits de Base/ APROMA, $64 \mathrm{p}$.

AOAC. 1997. Method 923.03. In : Official method analysis, $16^{\text {th }}$ Edition. Software Adobe and 
E - DOC/CJS. Washington, DC (USA). Association of Official Analytical Chemists International.

Asiedu J. J. 1989. Transformation des produits agricoles en zones tropicales. CTA KARTALA, France, Pays-bas, 113 - 1161.

Barel M. et M. Jacquet. 1994. La qualité du café : ses causes, son appréciation, son amélioration, Ed. France, vol.1, n¹ : 4 - 13.

Bernfeld P. 1955. Amylase alpha and beta. Methods in enzymology 1 S.P. Colswick and N.O.K., Ed. Academic Press Inc, New-York, $149-159$.

Cambroni H.R. 1989. Le caféier. Editions Maisonneuve et Larose, Paris, $166 \mathrm{p}$.

Carelli M. L. C., Lopes C. R. and L. C. Monaco. 1974. Turrialba, 24(4) : 398 - 401.

Charrier A. et J. Berthaud. 1975. Café, Cacao, Thé. Collection Production Horticole, CABI Publishing, 19, 251 - 264.

Clarke R. J. and L. J. Walker. 1974. J. Sci. Fd Agric., $25: 1389$ - 1404.

Claudine C. et D. R. Alexandre . 2006. Acides chlorogéniques pour ou contre? Sciences au Sud - le journal de l'IRD - $\mathrm{N}^{\circ} 35,11 \mathrm{p}$.

Clifford M. N. 1985. Chlorogenic acids. In Clarke, R. J. and Macrae, R. (Eds.). : Coffee Chemistry, Vol 1, Elsevier Applied Science Publishers, London and New-York : 153 202.

Coste R. 1989. Caféiers et Cafés dans le monde, Ed. GP. Maisonneuve et Larose, 11 p.

Coutouly G., E. Klein, E. Meyer. 1991. Travaux dirigés de biochimie. $2^{\mathrm{e}}$ édition, Doin (Eds.)., Paris, France, 279 p.

Frankenhauser M. 1986. A psychobiological framework for research on human stress and coping. In : Apple M. H. et Trumbell R. (Eds.). Dynamics of stress, Plenum Press, New York, $101 \mathrm{p}$.

Guyot B., Davrieux F., Manez J. C. et J. C. Vincent. 1993. Détermination de la caféine et de la matière sèche par spectrométrie proche infrarouge : application au café robusta et aux cafés torréfiés. Café, Cacao, Thé, vol. $37, n^{\circ} 1: 53-64$.

Guyot B., Picard H. et J. C. Vincent. 1984. Etude des composés stéroliques du café ( $C$. canephora). Café, Cacao, Thé, Paris, vol $28(4)$ : $47-62$.
Haslam E. 1981. In Biochemistry of Plants : A comprehensive Treatise, Vol 7, Secondary Plant Products, Ed. P. K. Stumpf and E. E. Conn, Academic Press, London, 527 - 556

Hulme, A.C., Rhodes, M. J. C., Gaillard, T. and L. S. C., Wooltorton. 1968. PI. Physiol. 43, $1154-1161$

Jagoret P. et F. Descroix. 2002. Recherche et Caféiculture, Montpellier : Cirad-CP, pp $44-59$.

Langler J. E., Libbey L. M. and E. A. Day. 1967. J. agric.Fd Chem., $15: 386$ - 391.

Mayer H. G. 1981. Composition of lipids of green coffee. Kaffee, Paul Parey, Hamburg, 21.

Perriot J. J. et R. Fabienne. 2006. L'analyse sensorielle du café : un outil pour la filière, des producteurs aux torréfacteurs. In : Recherche caféiculture. Montpellier: CIRAD-cp, 13 - 16.

Picard H., Guyot B. et J. C. Vincent. 1984. Etude des composés stéroliques de l'huile de café (Coffea canephora) Café, Cacao, Thé, vol. XXVIII, n¹, $4762 \mathrm{p}$.

Smith A. W. 1985. Introduction : In Clarke, R. J. and Macrae, R. (Eds.). : Coffee Chemistry, Vol 1, Elsevier Applied Science Publishers, London and NewYork : 1-4.

Svilaas A. 2004. Intakes of Antioxidants in Coffee, Wine and Vegetables are correlated with plasma carotenoids in Humans. Journal of Nutrition, Vol 134, 562 - 567.

Touré F. 2004. Etude de l'effet de l'association culturale du café robusta avec les légumineuses arborées sur les propriétés chimiques du café vert. DEA des Sciences et Technologies des Aliments. CNRA, Abidjan, $56 \mathrm{p}$.

Trugo L. C. 1985. Carbohydrates. In Clarke, R. J. and Macrae, R. (Eds.). : Coffee Chemistry, Vol 1, Elsevier Applied Science Publishers, London and New-York : 83 - 114.

Trugo L. C., Macrae R. and J. J. Dick. 1983. Sci. Fd Agric., 34, 300 - 306.

Voilley A., Sauvageot F. and D. Durand. 1977. Proc. $8^{\text {th }}$ Coll. ASIC, 251 - 259.

Warburton D. M. 1995. The effects of caffeine on cognition and mood without caffeine abstinence. Psychopharmacology, 119 : $475-492$. 